\title{
The Effect of Nutritional Supplementation with Fish Oil on Rankl/Opg Ratio in Gingival Crevicular Fluid of Patients Suffering From Chronic Periodontitis. A Randomized Controlled Trial
}

\author{
Dr.Debopriya Chatterjee ${ }^{1}$, Dr Mohamed Faizuddin ${ }^{2}$, Dr. Hemalata ${ }^{3}$, \\ Dr.Aishwarya Chatterjee ${ }^{4}$ \\ ${ }^{1}$ (Department of Dentistry,IQ City Medical College, Durgapur, West Bengal,India) \\ ${ }_{2}^{2}$ (Department of Periodontics, M.R.Ambedkar Dental College \& Hospital, Bangalore, India) \\ ${ }^{3}$ (Department of Periodontics, M.R.Ambedkar Dental College \& Hospital, Bangalore, India) \\ ${ }^{4}$ (Department of Maxillofacial Surgery, Ramakrishna Mission Sevaprathisthan, Kolkata, West Bengal)
}

\begin{abstract}
Fish oil, which is rich in omega-3 polyunsaturated fatty acids, has been shown to exert anti inflammatory influence in animal models and in many inflammatory diseases. The present study was designed to study the effect of nutritional supplementation with fish oil on RANKL/OPG ratio in gingival crevicular fluid of patients suffering from chronic periodontitis A total 60 subjects participated in the study and were randomly divided into experimental and control group consisting 30 each. The experimental group received nutritional supplementation with $300 \mathrm{mg}$ of fish oil capsule containing $30 \mathrm{mg}$ of DHA (Docosahexanoic acid) $20 \mathrm{mg}$ of EPA (Eicosapentanoic acid). The control group received placebo consisting gelatin capsule and starch. GCF samples were harvested from all the subjects and samples were stored and analyzed for RANKL and OPG levels using commercially. Our investigation revealed that the baseline RANKL/OPG ratio between the two groups was almost same. In both the groups after 6 weeks follow up there was a statistically significant decrease in the RANKL/OPG ratio. Comparing their mean RANKL/OPG ratio after 6 weeks there was a difference in their means with greater reduction in the experimental group. However this difference was not statistically significant. Fish oil may play a role as an inflammatory in periodontal diseases.
\end{abstract}

Key Words: Fish oil, RANKL/OPG

\section{Introduction}

Periodontitis is an inflammatory disease which results from complex interactions between plaque micro organisms and host immune system ${ }^{1}$. Various inflammatory mediators like arachidonic acid metabolites and cytokines are released during host defence in periodontal infection to counteract the bacteria ${ }^{2}$. Although the periodontal diseases are associated with specific pathogens, the research over the last 2 decades has revealed that most of the tissue damage is caused by the host response to infection and not by the infectious agent's directly ${ }^{3}$.

Evidence from various animal models and clinical studies has reported that omega-3 fatty acids have a beneficial effect in inflammatory diseases ${ }^{4}$.Omega -3 is polyunsaturated fatty acids are known to lessen the severity and minimize symptoms of chronic inflammatory diseases, including rheumatoid arthritis and inflammatory bowel disease 5 .

Periodontitis is an inflammatory disease and a Japanese longitudinal study has reported that fish oil which has an anti-inflammatory action may benefit periodontal health. According to this study fish intake was positively associated with intake of polyunsaturated fatty acids and therefore intake of fish may contribute in protecting against periodontal disease progression in elderly subjects ${ }^{6}$.

Periodontitis is an inflammatory disease and is characterized by destruction of supporting tissue and resorption of alveolar bone. RANK-RANKL and OPG axis is reported to be involved in bone metabolism and in periodontitis RANKL /OPG ratio is considered as a reliable marker of bone resorption ${ }^{7}$. The present randomized control trial is designed to study the effect of nutritional supplementation of fish oil on alveolar bone resorption in cases of chronic periodontitis by estimating the RANKL/OPG ratio in GCF in the patients suffering from chronic periodontitis. 


\section{Study Population}

\section{Method}

The present study was conducted in the Department of Periodontics, M. R. Ambedkar Dental College, and Hospital, Bangalore, Karnataka. After obtaining ethical clearance from institutional board and ethical committee of M.R. Ambedkar Dental College and Hospital, 256 patients visiting the department of periodontics were contacted. Aims, objectives and methodology were explained .Out of 256, 107 agreed to participate and signed the consent form. 60 of these patients met the inclusion criteria and they were divided randomly into experimental and control group. The randomization was done by lottery method. The nature and purpose of the study were explained to the subjects, and written consent was taken. The GCF samples were obtained from each of the subjects by placing colour coded, calibrated, volumetric, micro capillary pipettes with $0-5 \mu 1$ range. Volumetric micropipettes were placed extra-crevicularly at the entrance of the gingival crevice and a standardized volume of $3 \mu \mathrm{l}$ each was collected for estimation of RANKL and OPG.

\section{Selection Criteria Inclusion criteria}

1. Non-vegetarian who do not include fish in their diet frequently

2. Those who agreed not to eat fish and fish products ( 3 weeks before and during the study period)

3. Patients suffering from chronic periodontitis

4. Bleeding on probing

5. Probing pocket depth $\geq 5 \mathrm{~mm}$

6. Clinical attachment loss $\geq 3 \mathrm{~mm}$

7. Patient above age $\geq 35$ years old

8. Number of remaining teeth $\geq 20$ excluding third molar

\section{Exclusion Criteria}

1. History of underlying systemic disease

2. History of Periodontal treatment in past 6 months

3. Under antibiotic/NSAIDS treatment during past 6 months

4. History of Tobacco chewing and current or former smoker

5. Pregnant and lactating female.

6. Patients on steroid therapy and oral contraceptives

7. Any medication affecting periodontal health.

8. Patients allergic to fish and fish product.

Group I (Experimental group): 30 subjects, who received scaling and root planning and 300mg of fish oil supplements in form of capsule, thrice a day.

Each capsule contained 300mg fish oil:

- $\quad 30 \mathrm{~g}$ of DHA(Docosahexanoic acid)

- $20 \mathrm{mg}$ of EPA(Eicosapentanoic acid)

Group II (Control group): 30 subjects who received scaling and root planning and placebo thrice a day. Placebo consisted of gelatin capsule containing starch.

Of 30 subjects in each group, 27 subjects returned after 6 week for follow up. 3 subjects in each group failed to return for follow up.

pocket depth was defined as the distance from the free gingival margin to the bottom of the pocket or the sulcus and clinical attachment level was defined as the distance from the cemento-enamel junction to the bottom of the pocket or sulcus. The gingival crevicular fluid samples were collected using the micro capillary pipettes.

\section{Method Of Collection Of Gcf:}

The subjects were asked to rinse their mouth vigorously with water to cleanse the teeth of loosely adherent debris. Samples of GCF were obtained from the sites by placing colour coded, calibrated, volumetric, micro capillary pipettes with $0-5 \mu 1$ range ${ }^{a}$. Site with deepest probing depth was selected and isolated with cotton rolls. Volumetric micropipettes were placed extra crevicularly at the entrance of the gingival crevice, and the GCF samples were collected from each patient. The pipettes contaminated with blood or saliva was discarded. The pipette with collected fluid was wrapped in a sterile aluminium foil to prevent oxidation, placed in a plastic vial and immediately stored at $-700 \mathrm{C}$, until analyzed for $\mathrm{OPG}^{\mathrm{b}}$ and RANKL ${ }^{\mathrm{c}}$ with commercially available ELISA kits. 


\section{Statistics}

Mean and standard errors were calculated. The Statistical software namely statistical package for social science version 17 used for the analysis of the data and Microsoft word and Excel have been used to generate graphs, tables. Student t test (two tailed, independent) has been used to find the significance of study parameters on continuous scale between two groups Inter group analysis on metric parameters. Nonparametric tests namely Wilcoxons-signed rank test and Mann Whitney U test were used for testing the statistical significance. The significance level was set a $P>0.05$.

\section{Results}

Among 30 subjects in the experimental group, three subjects were excluded because they did not follow up. In the control group also three subjects were lost. So final of 54 subjects (29 male and 25 female) in the age range of 35-60 yrs who were eligible and willing to participate were included in the study. The mean levels of RANKL and OPG were estimated in the gingival crevicular fluid. There was no statistically significant difference in the gender and age distribution among the experimental and control group.

The mean RANKL/OPG ratio in experimental group at baseline was $63.70 \pm 81.98 \mathrm{pg} / \mathrm{ml}$ and that of the control group was $61.11 \pm 85.91 \mathrm{pg} / \mathrm{ml}$. There was no statistically significant difference in the RANKL/OPG ratio between the experimental group and control group $(\mathrm{p}=0.634)$ at the baseline. The mean RANKL/OPG ratio in experimental group was $30.23 \pm 48.51 \mathrm{pg} / \mathrm{ml}$ and control group was $43.24 \pm 52.99 \mathrm{pg} / \mathrm{ml}$ after 6 weeks .There was a difference in their means after 6 weeks but was not statistically significant ( $\mathrm{p}=0.058)$. (Table 1 , Table 2, Table 3, Table 4 Fig.1)

In the control group there was reduction in the RANKL/OPG ratio from baseline to after 6 weeks. This difference was statistically significant $(p=0.001)$. Similarly even in the experimental group there was a significant reduction in the RANKL/OPG ratio from baseline to 6 weeks after treatment, this reduction was statistically significant $(\mathrm{p}=0.001)$. When the means of RANKL/OPG ratio after 6 weeks of experimental and control group were compared, there was a considerable reduction in the ratio in experimental group compared to control group. However this difference was not statistically significant.

\section{Figures And Tables}

Table 1: Comparison between the baselines and after 6 weeks values of RANKL/OPG ratio within the experimental group

\begin{tabular}{|c|c|c|c|}
\hline & $\mathrm{N}$ & Mean & $\mathrm{p}$ \\
\hline Baseline & 27 & $63.70 \pm 81.98$ & 0.001 \\
6 week & 27 & $30.23 \pm 48.51$ & \\
\hline
\end{tabular}

Table 2: Comparison between the baseline and 6 weeks values of RANKL/OPG ratio within the control group

\begin{tabular}{|c|c|c|c|}
\hline & $\mathrm{N}$ & Mean & P value \\
\hline Baseline & 27 & $61.11 \pm 85.91$ & 0.001 \\
6 weeks & 27 & $43.24 \pm 52.99$ & \\
\hline
\end{tabular}

Table 3: Comparison between the RANKL/OPG ratio values of experimental group and control group at the baseline

\begin{tabular}{|c|c|c|c|}
\hline Groups & $\mathrm{N}$ & Mean & P Value \\
\hline Experimental & 27 & $63.70 \pm 81.98$ & \\
control & 27 & $61.11 \pm 85.91$ & 0.634 \\
\hline
\end{tabular}

Table 4: Comparison between the RANKL/OPG ratio values of experimental group and control group after 6 weeks

\begin{tabular}{|c|c|c|c|}
\hline Groups & $\mathrm{N}$ & Mean & P Value \\
\hline Experimental & 27 & $30.23 \pm 48.51$ & 0.058 \\
Placebo & 27 & $43.24 \pm 52.99$ & \\
\hline
\end{tabular}




\section{\begin{tabular}{|l|l|l}
\hline & 1 & $\mid$
\end{tabular}}

Figure 1: Comparison of RANKL/OPG ratio between experimental group and control group:

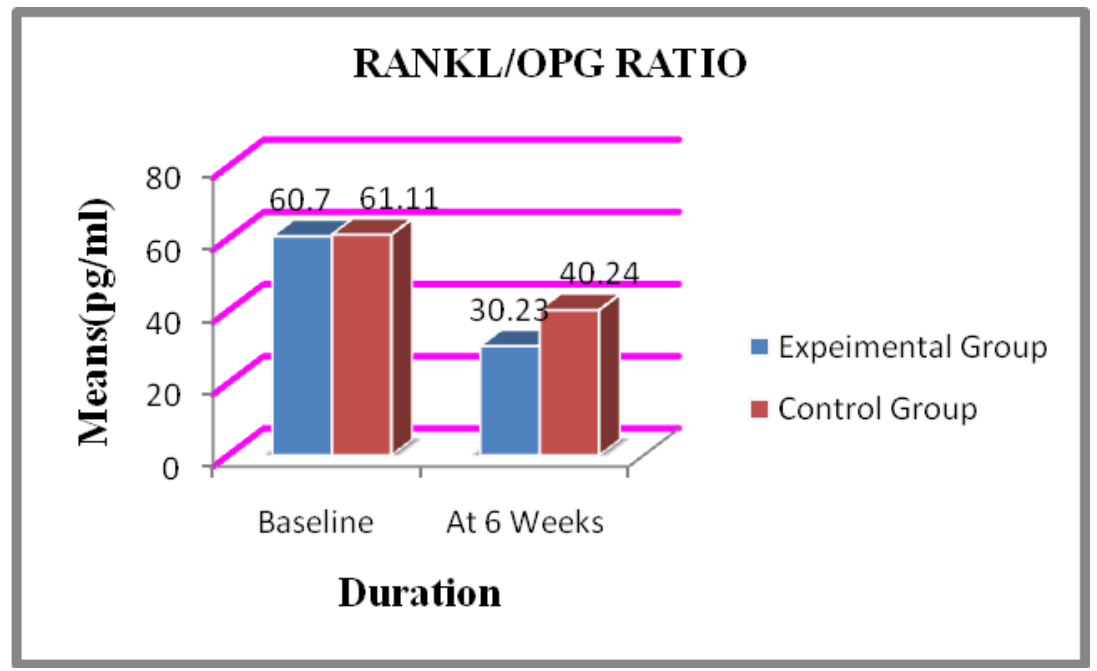

\section{Discussion}

Periodontitis is a complex disease in which disease expression involves intricate interactions of the pathogens with the host immune inflammatory response and subsequent alteration in the bone and connective tissue homeostasis ${ }^{8}$.

The initial response to bacterial infection is a local inflammatory reaction that activates the innate immune system. Amplification of these initial localized responses results in the release of an array of cytokines and other mediators and propagation of inflammation through the gingival tissues. The failure to encapsulate the inflammatory front, within gingival tissue, results in expansion of the response to adjacent alveolar bone ${ }^{9}$. This inflammatory process then drives the destruction of connective tissue and alveolar bone that is the cardinal sign of periodontitis.

One of the pathways involved in periodontal disease pathogenesis is the synthesis and release of prostaglandins and other arachidonic acid metabolites within periodontal tissues ${ }^{10}$. Both bacterial and host factors initiate tissue damage. This damage allows phospholipids in plasma membranes of cells to become available for action by phospholipase A2 and thereby results in production of free arachidonic acid (AA). AA can be metabolized via the cyclooxygenase (CO) or lipoxygenase (LO) pathways. Two isoforms of cyclooxygenase are recognized. Cyclooxygenase $1(\mathrm{COX}-1)$ is constitutively expressed and is important for physiologic functions including gastric cytoprotection. Cyclooxygenase $2(\mathrm{COX}-2)$ is inducible, upregulated by proinflammatory cytokines, and thought to be involved in inflammation ${ }^{11}$.

In the recent decades host modulation has been attempted as treatment modality for periodontal disease. One of the emerging host modulator is Omega-3 fatty acid. The first evidence of the important role of dietary intake of omega-3 polyunsaturated fatty acids (PUFAs) in inflammation was derived from epidemiological observations of the low incidence of autoimmune and inflammatory disorders such as psoriasis, asthma and type-1 diabetes as well as the complete absence of multiple sclerosis, in a population of Greenland Eskimos $^{12}$.

Studies have shown that increased consumption of these fatty acids results in their incorporation into inflammatory cell phospholipids. The decreased amounts of AA mean a decreased amount of substrate available for synthesis of AA-derived eicosanoids. Thus feeding fish oil to laboratory rodents or supplementing the diet of humans with fish oil has been reported to result in decreased production of a range of eicosanoids including PGE2, thromboxane B2, LTB4, 5-hydroxyeicosatetaenoic acid and LTE4 by inflammatory cells ${ }^{13,14}$.

In addition EPA is also able to act as a substrate for both cyclooxygenase and 5-lipoxygenase, giving rise to eicosanoids with a slightly different structure to those formed from arachidonic acid ${ }^{15,16}$.

Hence based on these evidence the present study was designed.

Periodontitis is an inflammatory disease of supporting tissues of teeth and is characterized by resorption of alveolar bone. RANK-RANKL and OPG axis is reported to involve in bone metabolism in periodontitis and RANKL /OPG ratio is considered as a reliable marker of bone resorption7. Receptor activator of NF- $\mathrm{KB}$ ligand (RANKL), a member of the Tumor Necrosis Factor (TNF) ligand superfamily, was identified as a cell membrane- bound factor responsible for stimulation of osteoclast differentiation and bone resoprtion ${ }^{17,18}$. RANKL is produced as a membrane-bound or secreted ligand by osteoblasts, fibroblasts, or activated T- and B-cells. 
By activating its cognate RANK receptor on the surface of pre-osteoclasts, it triggers their fusion and differentiation into mature osteoclasts, thus activating bone resorption . The action of RANKL can be blocked by its soluble decoy receptor osteoprotegerin (OPG), which is a member of the TNF receptor superfamily, with structural homology to RANK .

By binding to RANKL, OPG prevents its further interaction with RANK, and subsequently all the down-stream molecular events that lead to osteoclast differentiation and bone resorption.

Increased RANKL or decreased OPG local expression can cause bone resorption at various sites of the human skeleton. Conversely, decreased RANKL or increased OPG expression could result in enhanced bone formation .Hence, a number of clinical studies have investigated the concentrations of RANKL and OPG in gingival tissues or crevicular fluid extracted from individuals with chronic periodontitis to determine the RANKL/OPG ratio ${ }^{19}$.

In the present study, 60 subjects with chronic periodontitis were recruited and divided into two groups consisting of 30 subjects' each. Group I (Experimental group) consisted 30 subjects, who received scaling and root planning and $300 \mathrm{mg}$ of fish oil capsule supplements, thrice a day. Group II (Control group) consisted 30 subjects who received scaling and root planning and placebo thrice a day. After 6 weeks, the base line parameters were re-recorded and a comparison was made between group I and group II. Of 30 subjects in each group, 27 subjects returned after 6 week for follow up. 3 subjects in each group failed to return for follow up. We have taken patients who were non fish eater or abstain from fish eating since last 3 weeks and during the study period.

6 week time interval was decided based on a study by Marangoni $\mathrm{F}$ et al in which eight normal volunteers (four men and four women) were treated with $3 \times 1 \mathrm{~g}$ capsules of n-3 fatty acid ethyl esters for a period of 18 weeks. Fatty acids of plasma, platelets, monocytes and red blood cells were analyzed at $0,6,12$ and 18 weeks of treatment. During treatment, accumulation of EPA in plasma and cells was almost maximal at 6 weeks, whereas that of DHA reached a peak at 18 weeks. Arachidonic acid declined somewhat at 12 weeks in plasma and more markedly at 18 weeks in red blood cells and monocytes ${ }^{20}$.

The RANKL/OPG ratio has a potential diagnostic value for untreated periodontitis. The result of our study has revealed that the mean RANKL/OPG ratio in experimental group who received scaling and root planing along with nutritional supplementation of fish oil at baseline was $63.70 \pm 81.98 \mathrm{pg} / \mathrm{ml}$ and after 6 weeks follow up was $30.23 \pm 48.51 \mathrm{pg} / \mathrm{ml} .(\mathrm{p}=0.001)$. In the control group who received scaling and root planing along with placebo the mean RANKL/OPG at baseline was $61.11 \pm 85.91 \mathrm{pg} / \mathrm{ml}$ and after 6 weeks follow up was $43.24 \pm 52.99 \mathrm{pg} / \mathrm{ml} .(\mathrm{p}=0.001)$.

In both the groups there was a statistically significant decrease in the RANKL/OPG ratio. In the control group this decrease in the RANKL/OPG ratio could be due to the mechanical therapy. At the baseline RANKL/OPG ratio in the experimental group and control group was $63.70 \pm 81.98 \mathrm{pg} / \mathrm{ml}$ and $61.11 \pm 85.91 \mathrm{pg} / \mathrm{ml}$ $(\mathrm{p}=0.634)$ respectively, there was no statistically significant difference between the two groups at the baseline $(\mathrm{p}=0.634)$. After 6 weeks the RANKL/OPG ratio in the experimental group and control group was $30.23 \pm 48.51 \mathrm{pg} / \mathrm{ml}$ and $43.24 \pm 52.99 \mathrm{pg} / \mathrm{ml}$ respectively. Comparing their means after 6 weeks there was a difference in their means but which was not statistically significant $(\mathrm{p}=0.058)$.In the experimental group this reduction is due to the adjunct use of fish oil which has anti-inflammatory properties, along with scaling and root planing.

\section{Conclusion}

The results of our study indicates that nutritional supplementation of fish oil along with scaling and root planing in the experimental group did reduce RANKL/OPG ratio compared to scaling and root planing in the control group. This may form the basis for the adjunct use of fish oil therapy in periodontal disease in the future.

\section{References}

[1]. Page RC,Korman KS. The pathogenesis of human periodontitis: An introduction. Periodontol 2000 1997; 14:9-11.

[2]. Howell TH, Williams RC. Nonsteroidal anti-inflammatory drugs as inhibitors of periodontal disease progression. Crit Rev Oral Biol Med 1993;4:177-196.

[3]. Hesham EL-Sharkawy, Nayer Aboelsaad, Mohamed Eliwa, Mohmoud Darweesh, Mohammad Alshahat, Alpdogan Kantarci. Adjunctive Treatment of Chronic Periodontitis with Daily Dietary Supplementation with Omega-3 Fatty Acids and Low-Dose Aspirin. J Periodontol 2010;81:1635-1643

[4]. Penny M. Kris-Etherton, William S. Harris, Lawrence J. Appel. Fish Consumption, Fish Oil, Omega-3 Fatty Acids, and Cardiovascular Disease. Circulation.2002; 106:2747-2757.

[5]. Bruce A Watkins, Yong Li, Hugh E.Lippman, Shulin Feng. Modulatory Effect of omega-3 polyunsaturated fatty acids on osteoblast function and bone metabolism. Prostaglandin, Leukotrienes and Essential Fatty Acids 2003; 68:387-398.

[6]. Masanori Iwasaki, Akihiro Yoshihara , Paula Moynihan, ReikoWatanabe , George W. Taylor D.M.D., Dr.P.H. d, Hideo Miyazaki Ph.D. Longitudinal relationship between dietary u-3 fatty acids and periodontalDisease . Nutrition xxx 2009; 1-5.

[7]. David L. Cochran. Inflammation and Bone Loss in Periodontal Disease. J Periodontol 2008; 79:1569-1576.

[8]. . Offenbacher S, Barros SP, Singer RE, Moss K,Williams RC, Beck JD. J Periodontol 2007; 78:1911-1925. 
[9]. Graves DT, Cochran D. The contribution of interleukin- 1 and tumor necrosis factor to periodontal tissue destruction. J Periodontol 2003; 74:391-401.

[10]. Modulation of the host Response in periodontal Therapy. J Periodontol 2002; 73:460-470.

[11]. Howell TH, Williams RC. Non steroidal anti-inflammatory drugs as inhibitors of periodontal disease progression. Crit Rev Oral Med 1993; 4:177-196.

[12]. Williams RC, Jeffcoat MK,Howell TH et al. Altering the progression of human alveolar bone loss with non-steroidal antiinflammatory drug flubiprofen. J Periodontol 1989; 60:485-490.

[13]. R.I. Sperling, A.I. Benincaso, C.T. Knoell, et al., Dietary u-3 polyunsaturated fatty acids inhibits phosphoinositide formation and chemotaxis in neutrophils. J. Clin.Invest. 1993; 91: 651-660.

[14]. D. Rees, E.A. Miles, T. Banerjee, et al., Dose-related effects of eicosapentaenoic acid on innate immune function in healthy humans: a comparison of young and older men. Am. J. Clin. Nutrition 2006; 86:331-342.

[15]. Philip C. Calder Polyunsaturated fatty acids and inflammatory processes: New twists in an old tale. Biochimie 2009;91:791-795.

[16]. T.H. Lee, R.L. Hoover, J.D. Williams, et al., Effects of dietary enrichment with eicosapentaenoic acid and docosahexaenoic acid on in vitro neutrophil and monocyte leukotriene generation and neutrophil function. N. Eng. J. Med. 1985; 312: 1217-1224.

[17]. Lacey, D. L., Timms, E., Tan, H. L., Kelley, M. J., Dunstan, C. R., Burgess, T. et al. Osteoprotegerin ligand is a cytokine that regulates osteoclast differentiationand activation. Cell 1998; 93:165-176.

[18]. Kong, Y. Y., Yoshida, H., Sarosi, I., Tan, H. L., Timms, E., Capparelli, C.,et al. OPGL is a key regulator of osteoclastogenesis, lymphocyte development andmlymph-node organogenesis. Nature 1999; 397:315-323.

[19]. Belibasakis GN, Bostanci N. The RANKL-OPG system in clinical periodontology. J Clin Periodontol 2012; 39:239-248.

[20]. Weber PC, Leaf A: Cardiovascular effects of omega 3 fatty acids Atherosclerosis risk factor modification by omega 3 fatty acids. In Simopoulos AP, Kifer RR, Martin RE, Barlow SM (eds): "Health Effects of w3 Polyunsaturated Fatty Acids in Seafoods,".World Rev Nutr Diet. Basel Karger 1991; 66: 218-232. 